Pacific Journal of Mathematics

CLOSED AND IMAGE-CLOSED RELATIONS

PHi Frankie and R. H. SORGenfrey 


\section{CLOSED AND IMAGE-CLOSED RELATIONS}

\section{S. P. Franklin and R. H. Sorgenfrey}

If $X$ and $Y$ are topological spaces, a relation $T \leqq X \times Y$ is upper semi-continuous at the point $x$ of the domain $D(T)$ of $T$ if for each neighborhood $V$ of $T(x)$, there is a neighborhood $U$ of $x$ such that $T(U) \cong V$. Results so far published about such relations usually require that they be closed (as subsets of the product space) or image-closed $(T(x)$ is closed in $Y$ for each $x \in X)$. Given any relation $T$, it seems natural to consider the associated relations $T^{\prime}$ and $\bar{T}$, where $T^{\prime}$ is defined by $T^{\prime}(x)=\overline{T(x)}$ and $\bar{T}$ is the closure of $T$ in the product space. In particular, it is pertinent to ask under what conditions the upper semi-continuity of $T$ implies that of $T^{\prime}$ or $\bar{T}$, or that $T^{\prime \prime}=\bar{T}$. As might be expected, the answers to these questions take the form of restrictions on $Y$, and, indeed, serve to characterize regularity, normality, and compactness.

Other relation-theoretic characterizations have been given previously. In [6], Engelking characterizes regularity and compactness (in two ways), and in [10], Michael characterizes normality, collectionwise normality, perfect normality, and paracompactness. Ceder [1] characterizes $\mathfrak{m}$-compactness.

Terminology in this paper will follow Kelley [9]; in particular, regular and normal spaces need not be $T_{1}$. The following well known fact will be used: $T$ is upper semi-continucus (hereinafter abbreviated usc) on $D(T)$ if and only if the inverse under $T$ of each closed subset of $Y$ is closed in $D(T)$. A relation $T \subseteq X \times Y$ will be said to be on $X$ into $Y$ if and only if $D(T)=X$.

Statement of results. These are arranged so that for $n=1,2,3,4$, result $(2 n)$ is in the nature of a converse of result $(2 n-1)$, thus yielding the promised characterizations of regularity, normality, and various types of compactness.

(1) If $Y$ is regular and $T \subseteq X \times Y$ is usc at $x \in D(T)$, then $T^{\prime}(x)=\bar{T}(x)$.

Regularity of $Y$ does not imply the upper semi-continuity of $T^{\prime}$ or $\bar{T}$ for usc $T \subset X \times Y$ (see (6a) and (6b) below).

The statement of the next result, a converse of (1), and of several others will be expedited by a definition: Let $\Delta$ be a directed set and $p \notin \Delta$. Define a topology for $X=\Delta \cup\{p\}$ by letting each point of $\Delta$ be isolated and taking as a base at $p$ all sets of the form $S \cup\{p\}$ where 
$S$ is a final segment in $\Delta$. When equipped with this topology, $X$ will be called the net-space of $\Delta$. It is clear that each net-space has at most one accumulation point and therefore a rather simple structure.

(2) If for each net-space $X$ and usc $T$ on $X$ into $Y, T^{\prime}=\bar{T}$, then $Y$ is regular.

(3) If $Y$ is regular and $T \subseteq X \times Y$ is use and image-closed, then $T$ is closed in $D(T) \times Y$.

Under certain circumstances the hypothesis of regularity can be relaxed. A Fréchet space is one in which the closure of any subset $A$ is the set of all limits of sequences in $A$. Clearly, any first countable space is Fréchet, but the converse is not true (see [7]).

(3') Let $X$ and $Y$ be such that $X \times Y$ is a Fréchet space (e.g., $X$ and $Y$ first countable). If $Y$ is Hausdorff and $T \subset X \times Y$ is usc and image-closed, then $T$ is closed in $D(T) \times Y$.

(4) If for each net-space $X$ and usc image-closed relation $T$ on $X$ into $Y, T$ is closed, then either (a) $Y$ is regular, or (b) every closed nonregular subspace of $Y$ fails to be $R_{0}{ }^{1}$

The authors have been unable to remove the possibility (b) from the conclusion of this result. It is clear, however, that for $R_{0}$ (hence for $T_{1}$ )-spaces, (3) and (4) characterize regularity.

(5) If $Y$ is normal and $T \subseteq X \times Y$ is usc at $x \in D(T)$, then both $T^{\prime}$ and $\bar{T}$ are usc at $x$.

From (5) it is clear that if $Y$ is normal and $D(T)$ is closed, the upper semi-continuity of $T \leqq X \times Y$ implies that of $\bar{T}$. That this need not be the case if $D(T)$ is not closed is shown by the following

ExAmple. Let $X$ and $Y$ be the reals with usual topology and $f: Y \rightarrow X$ be defined by $f(y)=y^{-1}$ sin $y$ for $y \neq 0, f(0)=1$. Then $T=f^{-1} \backslash\{(0, y) \mid y \in Y\}$ is use on $D(T)$. However, $\bar{T}$ is not use at $0 \in D(\bar{T})$ since $V=\bigcup\{(n \pi-1, n \pi+1) \mid n$ an integer $\}$ is a neighborhood of $\bar{T}(0)$, but there is no neighborhood $U$ of 0 such that $T(U) \subseteq V$.

(6a) If for each net-space $X$ and usc relation $T$ on $X$ into $Y$, $T^{\prime}$ is usc, then $Y$ is normal.

\footnotetext{
${ }^{1}$ A space is $R_{0}$ if and only if point closures partition it. (Davis [4].)
} 
(6b) If $Y$ is Hausdorff and for each net-space $X$ and usc relation $T$ on $X$ into $Y, \bar{T}$ is use, then $Y$ is normal.

If $Y$ is infinite and equipped with the co-finite topology, ${ }^{2}$ then for every $X$ and usc $T$ on $X$ into $Y, \bar{T}$ is usc; hence the Hausdorff hypothesis in (6b) cannot be weakened even to $T_{1}$. Thus (5) and (6a) characterize normality, while (5) and (6b) characterize normality in the class of Hausdorff spaces.

Recall that for any infinite cardinal nt (defined as an initial ordinal) a topological space $Y$ is called m-compact if and only if each open cover of power $\leqq \mathfrak{m}$ has a finite subcover. Compact spaces are precisely those which are m-compact for each $m_{\text {. }} \boldsymbol{H}_{0}$-compact spaces are the countably compact spaces. m-compact spaces have been characterized in terms of the behavior of use relations on them by Ceder [1]. A space $X$ is said to have local weight $m$ if and only if $m$ is the least cardinal such that each point of $X$ has a basis of neighborhoods of power $\leqq m$. First countable spaces are those of local weight $\leqq \aleph_{0}$.

(7) If $Y$ is compact and $T \subseteq X \times Y$ is closed, then $T$ is usc on $D(T)$.

This result is well known and was apparently first noticed by Choquet [3].

(7m) If $X$ has local weight $\mathfrak{m}, Y$ is $\mathfrak{m}$-compact and $T \leqq X \times Y$ is closed, then $T$ is usc on $D(T)$.

(7\$.) If $X$ is first countable, $Y$ is countably compact and $T \subseteq X \times Y$ is closed. Then $T$ is usc on $D(T)$.

The corresponding results $\left(7^{\prime}\right),\left(7 \mathrm{~m}^{\prime}\right)$ and $\left(7 \mathbf{W}_{0}^{\prime}\right)$ about functions, in which the hypotheses on $X$ and $Y$ are the same and the conclusion is that every function $f: X \rightarrow Y$ with closed graph is continuous, are immediate corollaries. The net-space of an ordinal $\alpha$ will be denoted by $X_{\alpha}$.

(8) Let $Y$ be $T_{1}$. If for each net-space $X$ every closed $T$ on $X$ into $Y$ is usc, then $Y$ is compact.

(8m) Let $Y$ be $T_{1}$. If for each ordinal $\alpha \leqq \mathfrak{m}$, every closed 1 on $X_{\alpha}$ into $Y$ is usc, then $Y$ is n-compact.

\footnotetext{
2 i.e., the topology generated by the complements of finite sets.
} 
(8) Let $Y$ be $T_{1}$. If every closed $T$ on the sequence space $X_{\aleph_{0}}$ into $Y$ is usc, then $Y$ is countably compact.

These results are immediate consequence of the corresponding statements $\left(8^{\prime}\right),\left(8 \mathrm{~m}^{\prime}\right)$ and $\left(8 \mathbf{S}_{0}^{\prime}\right)$ in which it is hypothesized that each function $f$ from $X\left(X_{\mathfrak{m}}, X_{\boldsymbol{\aleph}_{0}}\right)$ into $Y$ with closed graph is continuous. If $Y$ is the set of natural numbers with the initial segments as a basis for the topology, then $Y$ is $T_{0}$ but not $T_{1}$, no function into $Y$ has closed graph, and $Y$ is not countably compact. Hence the $T_{1}$ hypothesis in $\left(8^{\prime}\right),\left(8 \mathfrak{m}^{\prime}\right)$ and $\left(8 \aleph_{0}^{\prime}\right)$ cannot be relaxed even to $T_{0}$. Clearly compactness ( $m$-compactness, countable compactness) in $T_{1}$ spaces is characterized by $(7)$ and $(8)\left((7 \mathfrak{m})\right.$ and $(8 \mathfrak{m}),\left(7 \boldsymbol{\aleph}_{0}\right)$ and $\left.\left(8 \boldsymbol{\aleph}_{0}\right)\right)$ as well as by their corresponding function results.

The hypothesis of first countability on $X$ in $\left(7 \boldsymbol{\aleph}_{0}\right)$ can be relaxed if the hypothesis on $Y$ is strengthened.

(9) If $X$ is a Hausdorff Fréchet space, $Y$ sequentially compact, and $T \subseteq X \times Y$ closed, then $T$ is usc on $D(T)$.

The corresponding function result $\left(9^{\prime}\right)$ is again an immediate corollary. One might hope for a converse to (9) patterned after $\left(8 \mathbf{S}_{0}\right)$, but the existence of compact, nonsequentially compact spaces (such as $\beta N$ ) makes the hope a vain one in view of (7).

Proofs of results. It will be convenient to give these in a somewhat different order from that of the statements.

Proof of (1). It is clear that for any relation, $T^{\prime}(x) \leqq \bar{T}(x)$. Suppose, therefore, that $y \in \bar{T}(x) \backslash T^{\prime}(x)$. Since $Y$ is regular and $T^{\prime}(x)$ is closed, there is a closed neighborhood $N$ of $T^{\prime}(x)$ not containing $y$. Since $T$ is use at $x$, there is an open neighborhood $U$ of $x$ such that $T(U) \subseteq N$. Then $U \times(Y \backslash N)$ is a neighborhood of $(x, y)$ not intersecting $T$, whence $(x, y) \notin \bar{T}$ or $y \notin \bar{T}(x)$.

Proof of (3). For all $x \in D(T), T(x)=\overline{T(x)}=T^{\prime}(x)$ by hypothesis and $T^{\prime}(x)=\bar{T}(x)$ by $(1)$.

Proof of $\left(3^{\prime}\right)$. Suppose there exist $x \in D(T)$ and $y \in Y$ such that $(x, y) \in \bar{T} \backslash T$. Since $X \times Y$ is Fréchet, there is a sequence $\left\{\left(x_{n}, y_{n}\right)\right\}$ in $T$ converging to $(x, y)$. Since $y \notin T(x)$, a closed set, and $\left\{y_{n}\right\} \rightarrow y$, there is an integer $k$ such that if $n>k, y_{n} \notin T(x)$. Thus $K=\left\{y_{n} \mid n>k\right\} \cup\{y\}$ and $T(x)$ are disjoint, and because $Y$ is Hausdorff, $K$ is closed. Since $T$ is usc, $T^{-1}(K)$ is closed in $D(T)$. But for $n>k, x_{n} \in T^{-1}(K)$ and $\left\{x_{n}\right\} \rightarrow x$, whence $x \in T^{-1}(K)$. Thus $T(x) \cap K \neq \varnothing$, a contradiction. 
Proof of (5). Let $E$ be either $T^{\prime}(x)$ or $\bar{T}(x)$, and let $V$ be a neighborhood of $E$. Since $E$ is closed and $Y$ is normal, there is a closed neighborhood $N$ of $E$ contained in $V$. Since $T$ is usc at $x$ and $N$ is a neighborhood of $T(x)$, there is an open neighborhood $U$ of $x$ such that $T(U) \subseteq N$. If $\bar{T}(U) \nsubseteq N$, there are $z \in U$ and $y \in Y \backslash N$ such that $(z, y) \in \bar{T}$. But $U \times(Y \backslash N)$ is a neighborhood of $(z, y)$ not intersecting $T$. Hence $T^{\prime}(U) \subseteq \bar{T}(U) \subseteq N \leqq V$, and both $T^{\prime}$ and $T$ are usc at $x$.

Proof of (6a). If $Y$ is not normal, there exist a closed $F \subset Y$ and a neighborhood $W$ of $F$ which contains no closed neighborhood of $F$. Direct the neighborhood system $\Delta$ of $F$ by $\subseteq$, and let $X=\Delta \cup\{p\}$ be the net-space of $\Delta$. Define $T$ on $X$ by $T(V)=V$ for all $V \in \Delta$, and $T(p)=F . \quad T$ is usc at $p$ (and hence on $X$ ) since for any neighborhood $V_{0}$ of $T(p)=F, U=\left\{V \in \Delta \mid V \cong V_{0}\right\} \cup\{p\}$ is a neighborhood of $p$, and $T(U) \subset V_{0}$. $T^{\prime}$, however, is not usc at $p$ since for each $V \in \Delta$, $T^{\prime}(V)=\bar{V}$ is a closed neighborhood of $F$ and hence is not contained in the neighborhood $W$ of $T(p)=F$.

Proof of (6b). Suppose $Y$ is not normal. We will construct a net space $X$ and usc $T$ on $X$ into $Y$ such that $T$ is not usc.

Case 1. $Y$ is regular. By (6a) there is a net-space $X$ and usc $T$ on $X$ into $Y$ such that $T^{\prime}$ is not usc. By (1), $T^{\prime}=\bar{T}$, and the construction is accomplished.

Case 2. $Y$ is not regular. There exist a closed $F \subset Y$ and $p \in Y \backslash F$ such that the closure of every neighborhood of $p$ intersects $F$. Let $\Delta$ be the family of all neighborhoods of $p$ which do not intersect $F$, direct $\Delta$ by $\subseteq$, and let $X=\Delta \cup\{p\}$ be the net-space of $\Delta$. Then $T$ defined on $X$ by $T(x)=x$ is usc.

We now show that $\bar{T}(p)=p$ : Let $p \neq q \in Y$. Since $Y$ is Hausdorff, there exist $V_{0} \in \Delta$ and a neighborhood $W$ of $q$ such that $W \cap V_{0}=\varnothing$. Then $U=\left\{V \in \Delta \mid V \subseteq V_{0}\right\} \cup\{p\}$ is a neighborhood of $p$ in $X$, hence $U \times W$ is a neighborhood of $(p, q)$ in $X \times Y$. If $(V, y) \in U \times W$, then $y \notin V=T(V)$ since $y \in W$ and $V \cap W \subseteq V_{0} \cap W=\varnothing$. Hence $(V, y) \notin T$, i.e., $(U \times W) \cap T=\varnothing$, whence $(p, q) \notin \bar{T}$, or $q \notin \bar{T}(p)$.

$\tilde{T}$ is not usc at $p$ since if $V \in \Delta, \bar{T}(V) \supset \bar{V}$ and is therefore not contained in the neighborhood $Y \backslash F$ of $p=\bar{T}(p)$.

Proof of (4). Assuming the proposition not true, there is a closed nonregular subspace $Z$ of $Y$ which is $R_{0}$. The existence of a net space $X$ and a nonclosed, image-closed, usc relation $T$ on $X$ into $Z$ will be 
demonstrated. Since $Z$ is closed, $T$, regarded as a relation on $X$ into $Y$ will have the same properties and provide the desired contradiction.

There exist closed $F \subset Z$ and $q \in Z \backslash F$ which do not have disjoint neighborhoods. Direct $\Delta=\{(V, W) \mid V$ is a neighborhood of $F$ and $W$ is a neighborhood of $q\}$ by $(V, W)>\left(V^{\prime}, W^{\prime}\right)$ if and only if $V \cong V^{\prime}$ and $W \cong W^{\prime}$, and let $X=\Delta \cup\{p\}$ be the net-space of $\Delta$. Define $T$ on $X$ into $Z$ by $T((V, W))=\left\{p_{V, W}\right\}^{-}$, where $p_{\nabla, W} \in V \cap W$, and $T(p)=F$. Then $T$ is image-closed; to show it usc at $p$, note that characteristic of $R_{0}$-spaces is the fact that $x \in O$, open, implies $\{x\}-\subset O$. Thus if $V_{0}$ is a neighborhood of $T(p)=F, U=\left\{(V, W) \mid(V, W)>\left(V_{0}, Y\right) \cup\{p\}\right.$ is a neighborhood of $p$, and $(V, W) \in U$ implies $p_{V, W} \in V \cap W \subset V_{0}$, whence $T((V, W))=\left\{p_{V, W}\right\}^{-} \subset V_{0}$. But the net $\left\{\left((V, W), p_{V, W}\right) \mid(V, W) \in \Delta\right\}$ in $T$ converges to $(p, q) \notin T$, and $T$ is not closed.

Proof of (2). Let $X$ be any net-space and $T$ be an image-closed usc relation on $X$ into $Y$. Then $T=T^{\prime}$ and, by hypothesis $T^{\prime}=\bar{T}$. Hence $T$ is closed and the hypothesis of (4) is satisfied. The present result will follow from (4) when it is shown that $Y$ (and hence every subspace of $Y$ ) is $R_{0}$. If this is not the case, there are points $q$ and $r$ of $Y$ such that $q \in(r)^{-}$but $r \notin\{q\}^{-}$. Let $X$ be the net-space consisting of a sequence $\left\{x_{n}\right\}$ and its limit $p$, and define $T$ on $X$ into $Y$ by $T\left(x_{n}\right)=\{q, r\} ; T(p)=\{q\}$. Since every neighborhood of $q$ contains $r, T$ is usc at $p$. But $r \notin T^{\prime}(p)=\{q\}^{-}$, while $r \in \bar{T}(p)$ since the sequence $\left\{\left(x_{n}, r\right)\right\}$ in $T$ converges to $(p, r)$. Hence $T^{\prime} \neq \bar{T}$.

Proof of $(7 \mathrm{~m})$. If $F$ is a closed subset of $Y, \pi_{Y}^{-1}(F) \cap T$ is closed in $X \times Y$. Since $Y$ is m-compact, $\pi_{x}$ is a closed mapping (Hanai [8]) and so $T^{-1}(F)=\pi_{X}\left(\pi_{Y}^{-1}(F) \cap T\right)$ is closed in $X$ and therefore in $D(T)$.

Proof of $\left(8 \mathfrak{n t}^{\prime}\right)$. If $Y$ is not $\mathfrak{m}$-compact, it follows from a lemma attributed to Chittenden [2] (see Ceder [1]), that there is an $\alpha$-net $\left\{y_{\beta}\right\}_{\beta<\alpha \leqq \mathfrak{m}}$ which has no cluster point. Define a function $f: X_{\alpha} \rightarrow Y$ by $f(\beta)=y_{\beta}$ if $\beta<\alpha$ and $f(p)=y_{0}$, where $y_{0}$ is an arbitrarily chosen point of $Y . \quad f$ is not continuous at $p$ since $\{\beta\}_{\beta<\alpha}$ converges to $p$ in $X_{\alpha}$ but $\{f(\beta)\}_{\beta<\alpha}=\left\{y_{\beta}\right\}_{\beta<\alpha}$, having no cluster point, cannot converge to $f(p)=y_{0}$ in $Y$.

Suppose $(x, y) \notin f$. If $x=\beta<\alpha$, then $y \neq y_{\beta}$. Let $W$ be any open neighborhood of $y$ not containing $y_{0}$. Then $(x, y) \in\{\beta\} \times W$, which is open and disjoint from $f$. If, on the other hand, $x=p$, then $y=y_{0}$. Since $y$ is not a cluster point of $\left\{y_{\beta}\right\}_{\beta<\alpha}$, there is an open neighborhood $U$ of $y$, not containing $y_{0}$, and a $\beta_{0}<\alpha$ such that $\beta \geqq \beta_{0}$ implies $y_{\beta} \notin U$. Let $N=\left\{\beta \mid \beta_{0} \leqq \beta<\alpha\right\} \cup\{p\}$. Then $(x, y) \in N \times U$ which is open and disjoint from $f$. 
(8') follows from $\left(8 \mathrm{~m}^{\prime}\right)$ since $Y$ is compact if and only if $Y$ is $\mathfrak{m}$ compact for all $\mathfrak{n}$ (Chittenden [2], Ceder [1]).

Proof of (9). Let $F$ be closed in $Y$. If $x_{0} \in \mathrm{cl}_{\mathcal{D}\left(T^{\prime}\right)} T^{-1}(F)$, there is a sequence $\left\{x_{n}\right\} \subseteq T^{-1}(F)$ converging to $x_{0}$ (since subspaces of Fréchet spaces are Fréchet [7]). For each $n$ choose $y_{n} \in T\left(x_{n}\right) \cap F$ and let $\left\{y_{n_{i}}\right\}$ be a subsequence of $\left\{y_{n}\right\}$ converging to $y_{0} \in Y$. But $y_{0} \in F$ and $\left\{\left(x_{n_{i}}, y_{n_{i}}\right)\right\}$ is contained in $T$ and converges to $\left(x_{0}, y_{0}\right)$. Thus, since $T$ is closed, $x_{0} \in T^{-1}(F)$.

\section{BIBLIOGRAPHY}

1. J. G. Ceder, Compactness and semi-continuous carriers, Proc. Amer. Math. Soc. 14 (1963), 991-3.

2. E. W. Chittenden, On general topology, Trans. Amer. Math. Soc. 31 (1929), 290-321.

3. G. Choquet, Convergences, Ann. Grenoble U. sec. Sci., Math. Phy. 23 (1947-48), $58-112$.

4. A. S. Davis, Indexed systems of neighborhoods for general topological spaces, Amer. Math. Monthly 68 (1961), 886-93.

5. J. M. Day and S. P. Franklin, Spaces of continuous relations (Math. Ann. to appear)

6. R. Engelking, Quelques rémarques concernant les operations sur les fonctions semicontinues dans les espaces topologiques, Bull. Acad. Polonaise Sci., ser. Sci. Math., Astr. Phys. 11 (1963), 719-26.

7. S. P. Franklin, Spaces in which sequences suffice, Fund. Math. 57 (1965), 107-16.

8. S. Hanai, Inverse images of closed mappings, I Proc Japan Acad. 37 (1961), 298304.

9. J. Kelley, General Topology, Princeton, 1955.

10. E. Michael, Continuous selections, I Annals of Math. 63 (1956) 361-82.

Receired August 13, 1965. 



\section{PACIFIC JOURNAL OF MATHEMATICS}

\section{EDITORS}

H. SAMELSON

Stanford University

Stanford, California

J. P. JANS

University of Washington

Seattle, Washington 98105

\section{J. DuGUNDJI}

University of Southern California

Los Angeles, California 90007

RICHARD ARENS

University of California

Los Angeles, California 90024

\section{ASSOCIATE EDITORS}

E. F. BECKENBACH
B. H. NEUMANN

F. WOLF

K. YoSIDA

\section{SUPPORTING INSTITUTIONS}

\author{
UNIVERSITY OF BRITISH COLUMBIA \\ CALIFORNIA INSTITUTE OF TECHNOLOGY \\ UNIVERSITY OF CALIFORNIA \\ MONTANA STATE UNIVERSITY \\ UNIVERSITY OF NEVADA \\ NEW MEXICO STATE UNIVERSITY \\ OREGON STATE UNIVERSITY \\ UNIVERSITY OF OREGON \\ OSAKA UNIVERSITY \\ UNIVERSITY OF SOUTHERN CALIFORNIA
}

\author{
STANFORD UNIVERSITY \\ UNIVERSITY OF TOKYO \\ UNIVERSITY OF UTAH \\ WASHINGTON STATE UNIVERSITY \\ UNIVERSITY OF WASHINGTON \\ AMERICAN MATHEMATICAL SOCIETY \\ CHEVRON RESEARCH CORPORATION \\ TRW SYSTEMS \\ NAVAL ORDNANCE TEST STATION
}

Mathematical papers intended for publication in the Pacific Journal of Mathematics should be typewritten (double spaced). The first paragraph or two must be capable of being used separately as a synopsis of the entire paper. It should not contain references to the bibliography. Manuscripts may be sent to any one of the four editors. All other communications to the editors should be addressed to the managing editor, Richard Arens at the University of California, Los Angeles, California 90024 .

50 reprints per author of each article are furnished free of charge; additional copies may be obtained at cost in multiples of 50 .

The Pacific Journal of Mathematics is published monthly. Effective with Volume 16 the price per volume (3 numbers) is $\$ 8.00$; single issues, $\$ 3.00$. Special price for current issues to individual faculty members of supporting institutions and to individual members of the American Mathematical Society: $\$ 4.00$ per volume; single issues $\$ 1.50$. Back numbers are available.

Subscriptions, orders for back numbers, and changes of address should be sent to Pacific Journal of Mathematics, 103 Highland Boulevard, Berkeley 8, California.

Printed at Kokusai Bunken Insatsusha (International Academic Printing Co., Ltd.), No. 6, 2-chome, Fujimi-cho, Chiyoda-ku, Tokyo, Japan.

\section{PUBLISHED BY PACIFIC JOURNAL OF MATHEMATICS, A NON-PROFIT CORPORATION}

The Supporting Institutions listed above contribute to the cost of publication of this Journal, but they are not owners or publishers and have no responsibility for its content or policies. 


\section{Pacific Journal of Mathematics

Vol. 19, No. $3 \quad$ July, 1966

S. J. Bernau, The spectral theorem for unbounded normal operators .......

Lu-san Chen, Asymptotic behavior of solutions of parabolic equations of

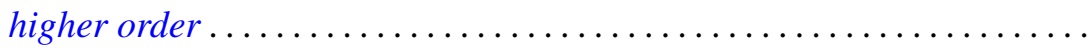

Lawrence William Conlon, An application of the Bott suspension map to the

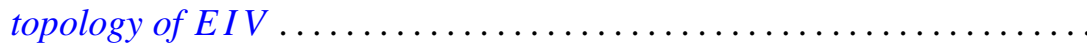

Neal Eugene Foland and John M. Marr, Sets with zero-dimensional kernels .........................................

Stanley Phillip Franklin and R. H. Sorgenfrey, Closed and image-closed

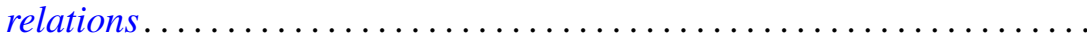

William Jesse Gray, A note on topological transformation groups with a

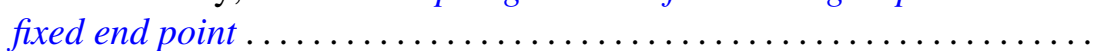

Myron Goldstein, $K$ - and L-kernels on an arbitrary Riemann surface ...... 449

George Joseph Kertz and Francis Regan, The exponential analogue of a generalized Weierstrass series .............................

Walter Leighton, On Liapunov functions with a single critical point ........ 467

Bernard Werner Levinger and Richard Steven Varga, On a problem of $O$.

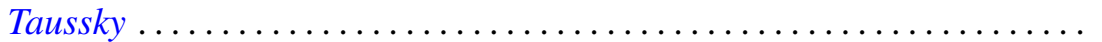

Lowell Duane Loveland, Tame subsets of spheres in $E^{3} \ldots \ldots \ldots \ldots \ldots . .489$

Erik Andrew Schreiner, Modular pairs in orthomodular lattices ......... 519

K. N. Srivastava, On dual series relations involving Laguerre polynomials ...............................

Arthur Steger, Diagonability of idempotent matrices.....

Walter Strauss, On continuity of functions with values in various Banach spaces...

Robert Vermes, On the zeros of a linear combination of polynomials ...

Elliot Carl Weinberg, On the scarcity of lattice-ordered matrix rings ....

Harold Widom, Toeplitz operators on $H_{p} \ldots \ldots \ldots \ldots \ldots$

Neal Zierler, On the lattice of closed subspaces of Hilbert space...

Irving Leonard Glicksberg, Correction to: "Maximal algebras and a theorem of Rado"

John Spurgeon Bradley, Correction to: "Adjoint quasi-differential operators of Euler type"

William Branham Jones, Erratum: "Duality and types of completeness in locally covex spaces".

Stanley P. Gudder, Erratum: "Uniqueness and existence properties of bounded observables" 